\title{
MACHINE PROTECTION FOR HIGH AVERAGE CURRENT LINACS
}

\author{
K. Jordan, T. Allison, J. Coleman, R. Evans, A. Grippo \\ Thomas Jefferson National Accelerator Facility, Newport News, VA 23606, USA
}

\begin{abstract}
A fully integrated Machine Protection System (MPS) is critical to efficient commissioning and safe operation of all high current accelerators. The Jefferson Lab FEL $[1,2]$ has multiple electron beam paths and many different types of diagnostic insertion devices. The MPS [3] needs to monitor both the status of these devices and the magnet settings which define the beam path. The matrix of these devices and beam paths are programmed into gate arrays, the output of the matrix is an allowable maximum average power limit. This power limit is enforced by the drive laser for the photocathode gun. The Beam Loss Monitors (BLMs), RF status, and laser safety system status are also inputs to the control matrix. There are 8 Machine Modes (electron path) and 8 Beam Modes (average power limits) that define the safe operating limits for the FEL. Combinations outside of this matrix are unsafe and the beam is inhibited. The power limits range from no beam to 2 megawatts of electron beam power. The timing requirements for the BLMs and $\mathrm{RF}$ is $\sim 1$ microsecond and for the insertion devices (viewers) is $\sim 1$ millisecond. The entire system is VME based and EPICS compatible. This paper describes the system as it is installed on the JLab $10 \mathrm{~kW}$ FEL.
\end{abstract}

\section{SYSTEM DESCRIPTION}

The driver accelerator for Jefferson Lab's Free Electron Laser will be capable of producing a 2 MWS electron beam (with the installation of the third cryomodule this fall). The machine can drive either an Infrared Laser (10 $\mathrm{kw} \mathrm{CW}$ ) or Ultraviolet Laser (1 kw CW). In addition to these two primary beam lines there are two diagnostic beam dumps and an insertable dump. There are also a variety of insertion devices that have different maximum average current limits. The MPS system was designed to minimize operator set-up time and confusion with choices of beam paths and power (and the possibility of mistakes). All of the devices that are required to properly accelerate beam and anything that can be inserted into the beam line or Laser is connected to the MPS system. The inputs to the system are organized first by speed (fast or slow) then by major system, and finally by location. The system response times depend on these inputs; the fast inputs are brought into the system on fiber optics, these respond in 1 microsecond to the absence of a $5 \mathrm{MHz}$ square wave on the fiber, the slow inputs $(<1$ millisecond) depend on a 24 Volt level for a permit (see adjacent table). Events like the BLM or RF system trips require immediate termination of the electron beam $(<10$ microsecond), where the dump water system tripping $(<1$ millisecond) is a bit more forgiving! These times are top level requirements and are not imposed by the MPS but rather the inputs in many cases are mechanical switches or relays.

There are three main components to the system; 1 . The Machine / Beam Mode Status Cards (MMSC \& BMSC), 2. The BLM VME cards, and 3. The Drive Laser Pulse Controller (DLPC).

\section{MACHINE AND BEAM MODES}

We have defined 8 Beam Modes and 8 Machine Modes. In both cases the Mode " 0 " is an invalid setup. Each of the MPS inputs fit into one of more these safe operating modes. The MPS uses magnet switch settings and currents to determine the Machine Modes (MM). The MMs are used internal to the MPS to enable the Drive Laser Pulse Controller (DLPC) which ultimately controls the average current of the electron beam. An example is when the beam viewer ITV3F04 is inserted the MPS sets the DLPC to Beam Mode (BM) 2. This is done by placing a $5 \mathrm{MHz}$ square wave on fiber number 1,2 , and 3 of the 8 fiber optic cables that connect the two systems together. The DLPC allows the operator to select combinations of micropulse frequency and macropulse frequency (or $\mathrm{CW}$ ) that are valid for the highest beam mode that is permitted. The DLPC assumes full bunch charge then calculates allowable combinations of micro and macro pulse width and frequency to conform to the allowable limit. An example is that one could run with this viewer in at 18 $\mathrm{MHz}$ micropulse frequency, 10 microsecond width, and $60 \mathrm{~Hz}$ repetition frequency, or at $4 \mathrm{MHz}$ micropulse frequency, 400 microsecond macropulse width, and at 2 $\mathrm{Hz}$ repetition rate. These are both used depending on operation at hand.

The Machine Mode Status Card (MMSC) takes up to 64 inputs from an optically isolated I/O card through the VME P2 back plane connector. These signals represent interlocks for systems such as the injector gun high voltage power supply status, magnet switch status, and magnet current status. These inputs are used to determine which machine modes are valid. This information is then passed to as many as four Beam Mode Status Cards (BMSC) that are physically the same as the MMSC but differ in functionality.

Each BMSC receives interlock information from systems such as vacuum valves, beam viewers, injector gun, beam dumps, and any other interlocks in the same fashion as the MMSC. They also accept the current valid machine mode from the MMSC through a front panel connector. Each BMSC then determines its allowable beam mode and passes this information to the Machine Protection Driver Card (MPDC).

The MPDC takes in the machine mode and beam mode data from the MMSC and BMSC modules through a front panel connector. The MPDC also monitors a fiber optic 
signal from the Fast Shutdown Summation Card that sums interlocks from systems such as RF Control Modules and Beam Loss Monitors. The MPDC then determines the maximum allowable beam mode and transmits this information to the Drive Laser Pulse Controller (DLPC) through 8 fiber optic lines.

All of the aforementioned VME cards use FPGA devices to interpret interlock inputs and determine valid machine and beam modes. The status of all the interlocks, allowable machine modes, and allowable beam modes are available to EPICS from each module through the VME back plane.

\section{Beam Modes}

The actual micropulse \& macropulse setting are determined by the DLPC

- Beam Mode 0, No Beam Allowed

- Beam Mode 1, Ceramic Viewer Mode

- Beam Mode 2, IR Beam Viewer Mode

- Beam Mode 3, UV Beam Viewer Mode

- Beam Mode 4, High Power Viewer Mode

- Beam Mode 5, IR Laser Alignment Mode

- Beam Mode 6, UV Laser Alignment Mode

- Beam Mode 7, Full Power Mode

Note that modes $4,5, \& 6$ can be used by the Laser Safety System to limit the Laser power

\section{Machine Modes}

The Beam Dump location is determined by monitoring the dipole power supply current and the magnet switch positions

- Machine Mode 0, No Beam Allowed; Magnet and Switch Settings Not Valid

- Machine Mode 1, Injector Dumplet

- Machine Mode 2, 2G Strait Ahead Dump

- Machine Mode 3, IR Recirculation Mode

- Machine Mode 4, UV Recirculation Mode

- Machine Mode 5, 6, 7, To Be Determined

\section{BEAM LOSS MONITORING SYSTEM}

It is easily understood that we don't want to trip-off the accelerator when using diagnostics since the beam loss produced by the short macropulse is not dangerous to the machine. This is why, in the past, we would have to mask the BLMs in before going into pulsed mode. (An action that must be performed by the operator or software and then the BLM system MUST be unmasked before going back to high power). This new VME based BLM system has the ability to "ignore" the loss detected during a macropulse of restricted size and duty factor. This results in a significant improvement to the systems performance. The new BLM cards (F0151) are configured by EPICS as to what macropulse widths to expect (firmware limited to less than 400us) and will also receive an appropriately delayed beam-sync signal from which to synchronize the window for which the electronics will "ignore" the detected beam loss that would otherwise trip the BLM channel. It is essential to state that after the time window in which the loss is ignored (or vetoed), the BLM interlocking circuit is forced to remain armed for a period of time that is 1250 times longer than the veto pulse. The factor of 1250 comes from the $0.08 \%$ duty factor restriction programmed into the firmware. (i.e. 400us * $1250=0.5 \mathrm{~s}$ or $2 \mathrm{~Hz}$ ) This "beam veto" signal cannot be tricked by a failure in the beam-sync source as the timing associated with it is calculated internal to the BLM fault circuit. The result is a system that allows insertion device operation with no procedural steps to ensure that it is done right. The BLM Beam-Veto function allows the operator to use insertion devices with no Interlock masking or changes in its native mode of operation. Finally, a consequence of a BLM system having only a single mode of operation as described above is that this mode of operation must be appropriate for running the machine in high current modes as well. In the normal setup for full power CW running, the system will continue to receive a beam-sync pulse at $60 \mathrm{~Hz}$ (or any rep-rate actually). In this case there would exist a short BLM veto pulse lasting approx 13us $(16.6 \mathrm{~ms} / 1250)$. This is consistent with a tolerated about of beam loss with a structure at $60 \mathrm{~Hz}$ of $0.08 \%$. The end result for $\mathrm{CW}$ running is that the BLM channel is armed to fault $99.92 \%$ of the time and that the $0.08 \%$ loss that is not seen is the loss that has been calculated as acceptable under the same line of logic used for Pulsed Mode.

All of the 12 channel BML boards are connected together with a common with a common clock that is initiated by a trip of any of the BLM channels. This clock initiated a shift register which latches the first three channel that trip thus recording the trip history.

\section{DRIVE LASER PULSE CONTROLLER}

The DLPC is responsible for enforcing the allowed Beam Mode. A block diagram of the system is show in figure 1. The heart of the system is a scalar board from ConOptics corporation. This has been fitted to a VME mother board and mounted in the VME crate. The laser is a commercial mode locked Nd:YLF that operates at the $20^{\text {th }}$ sub-harmonic of the $1497 \mathrm{MHz}$ RF frequency. Elector-optical (EO) cells are used to divide the $(\sim) 75$ $\mathrm{MHz}$ down by factors of 2 until the desired micropulse frequency is reached. This mode locker must be locked to the accelerator master oscillator for running beam (this is one of the beam mode 0 inputs).

Since the EO cells are not failsafe therefore the DLPC monitors the actual beam current and confirms the average power enabled by a given beam mode is not exceeded. The DLPC uses both the EO cells and a mechanical shutter to stop beam in the event of a fault. 


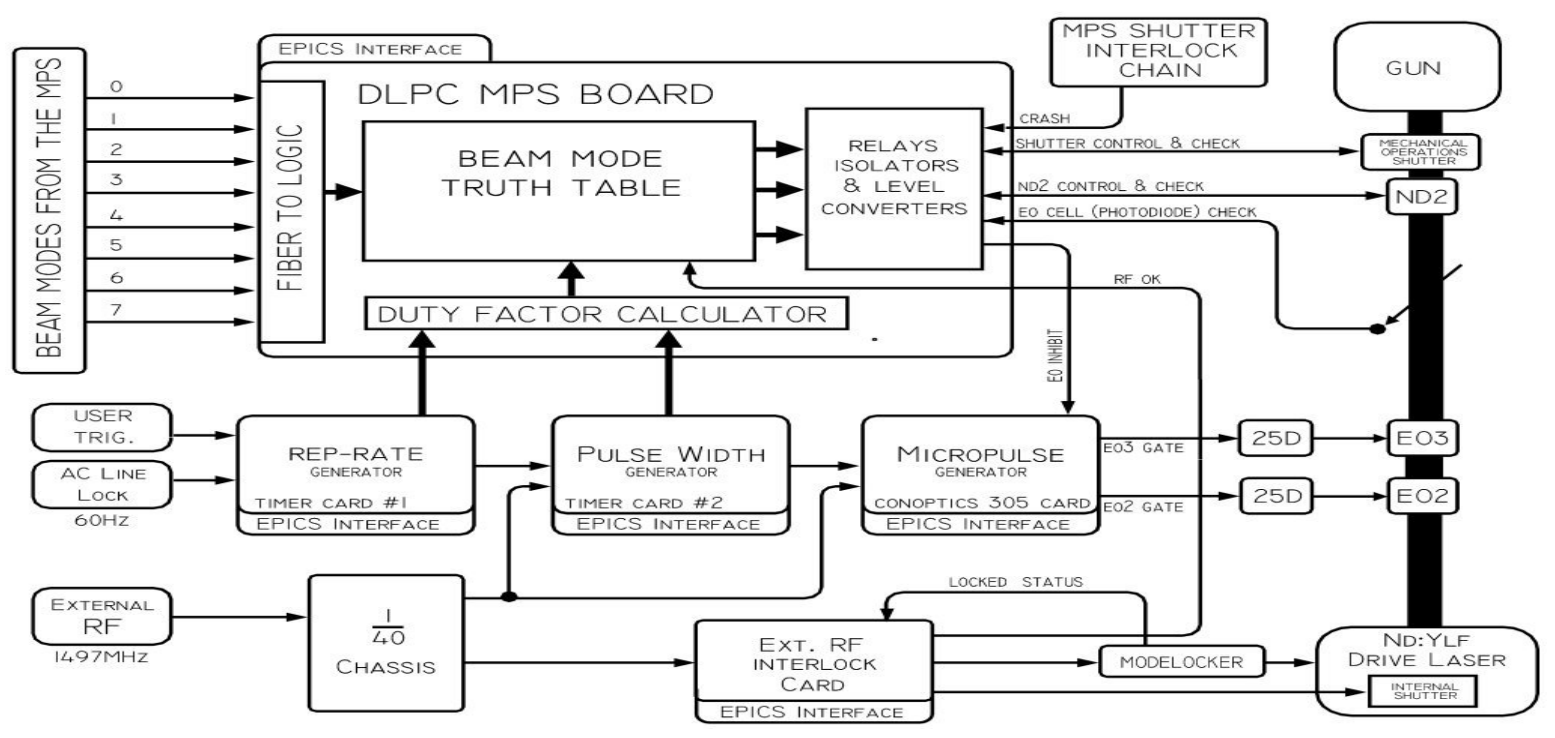

Figure 1: DLPC Block Diagram

\section{SCALABILITY}

The design which is based on a single FPGA control board, a common optically isolated input board and a fiber Input/Output board lends itself readily to support nearly any machine configuration.

The system described is configured for the MM/BM VME cards to reside next to each other in a single crate so the 25 pin "D" connector can be strapped with a ribbon cable. In the case one would have multiple locations in a larger facility each remote location would be connected in a serial fashion with 6 fiber optical cables from the 'last' to the 'next' with the final location connecting to the Drive Laser Pulse Controller. The system would able to seamlessly gather the information about the current configuration and status of insertion devices and pass it along to the final gate array which decides which Beam Mode to pass on to the DLPC. As always the status would be monitored in EPICS.

\section{CONCLUSIONS}

This system was designed with operability at the forefront of the design considerations. This was accomplished by the following key features; 1. BLM blanking - This allows for easy transition from full power mode to pulsed diagnostic mode, 2. Machine Modes allow for maintenance or commissioning of component in unused beam lines with out impacting operations and eliminates the need for masking of interlocks, 3. Post Mortem information is latched to identify first fault, 4. DLPC monitors actual beam current to confirm and enforce allowed beam mode, 5. All status is monitored through EPICS and available to operations staff.

In developing this system we had gone through an iteration with the previous JLab IR Demo machine.

This initial system consisted of VME boards borrowed from the Advanced Photon Source at Argonne National
Lab and a combination of Programmable Logic Controllers (PLCs) implemented by the CEBAF safety group headed by Kelly Mahoney. This system worked well to protect the machine but had limitations in getting the status of fault conditions cleanly back through EPICS and latching the first offender. This new system was designed to address these shortcomings as well as the challenges faced by an operational laser that may reach powers in excess of 100 kilowatts with planned follow on upgrades.

\section{ACKNOWLEDGEMENTS}

I would like to thank the members of the FEL team and in particular George Neil, Steve Benson and Michelle Shinn in providing motivation and insight to provide a system that both protects the accelerator and the laser and eases the operation and commissioning of the various systems. This work was supported primarily by the U.S. Dept. of Energy under contract DE-AC05-84-ER40150 (Thomas Jefferson National Accelerator Facility). The JLab FEL is supported by the Office of Naval Research, the Air Force Research Laboratory, the Commonwealth of Virginia and the Laser Processing Consortium.

\section{REFERENCES}

[1] G. R. Neil et al., Phys. Rev. Lett., 84, (2000) 662.

[2] S. V. Benson et al., "A $10 \mathrm{~kW}$ IRFEL Design for Jefferson Lab," PAC'01, Chicago, IL, June 2001, p. 249.

[3] http://laser.jlab.org 